\title{
Computationally Efficient DOA Tracking Algorithm in Monostatic MIMO Radar with Automatic Association
}

\author{
Huaxin Yu, ${ }^{1}$ Xiaofei Zhang, ${ }^{1,2}$ Xueqiang Chen, ${ }^{1}$ and Hailang $W u^{1}$ \\ ${ }^{1}$ College of Electronic and Information Engineering, Nanjing University of Aeronautics \& Astronautics, Nanjing 210016, China \\ ${ }^{2}$ Laboratory of Modern Acoustic of Ministry of Education, Nanjing University, Nanjing 210093, China
}

Correspondence should be addressed to Xiaofei Zhang; njxnd88@126.com

Received 16 March 2014; Revised 23 May 2014; Accepted 10 June 2014; Published 3 July 2014

Academic Editor: Frankie KitWing Chan

Copyright (c) 2014 Huaxin Yu et al. This is an open access article distributed under the Creative Commons Attribution License, which permits unrestricted use, distribution, and reproduction in any medium, provided the original work is properly cited.

We consider the problem of tracking the direction of arrivals (DOA) of multiple moving targets in monostatic multiple-input multiple-output (MIMO) radar. A low-complexity DOA tracking algorithm in monostatic MIMO radar is proposed. The proposed algorithm obtains DOA estimation via the difference between previous and current covariance matrix of the reduced-dimension transformation signal, and it reduces the computational complexity and realizes automatic association in DOA tracking. Error analysis and Cramér-Rao lower bound (CRLB) of DOA tracking are derived in the paper. The proposed algorithm not only can be regarded as an extension of array-signal-processing DOA tracking algorithm in (Zhang et al. (2008)), but also is an improved version of the DOA tracking algorithm in (Zhang et al. (2008)). Furthermore, the proposed algorithm has better DOA tracking performance than the DOA tracking algorithm in (Zhang et al. (2008)). The simulation results demonstrate effectiveness of the proposed algorithm. Our work provides the technical support for the practical application of MIMO radar.

\section{Introduction}

Multiple-input multiple-output (MIMO) radar employs multiple antennas to simultaneously transmit diverse waveforms and utilizes multiple antennas to receive the reflected signals [1-5]. Direction of arrival (DOA) algorithms in MIMO radar have been recently investigated in [6-24], which contain estimation of signal parameters via rotational invariance technique (ESPRIT) algorithms [6-8], Capon algorithms [10, 11], multiple signal classification (MUSIC) algorithms [9, 1215], parallel factor (PARAFAC) analysis algorithms [16-18], propagator method $[19,20]$, quaternion method [21, 22], compressive sampling methods [23, 24], and so on. However, the algorithms mentioned above are, generally, used in offline situation, and they are not applicable for tracking moving targets. The online algorithms can be used for real-time application of MIMO radar.

DOA tracking for array antenna has been investigated for a long time, which contains projection approximation and subspace tracking (PAST) algorithm [25], projection approximation and subspace tracking of deflation (PASTd) algorithm [26], Bi-iterative least-square method [27], Biiteration single value decomposition [28], and others [29, 30]. The DOA tracking algorithms in [25-30] have a high computational complexity. A low-complexity method was proposed in $[31,32]$ to track DOA of moving sources using the elements of the covariance matrix of the received signal in array signal processing, and the DOA tracking algorithm can implement automatic association, which is a key technique in DOA tracking [33].

DOA tracking for MIMO radar is to track the DOA of the moving targets. PARAFAC adaptive algorithms $[17,18]$ and PASTd [34] were used for DOA tracking for MIMO radar, but an extra data association is required. Kalman-PASTd was proposed in [35] for DOA tracking for monostatic MIMO Radar with automatic association. PARAFAC adaptive algorithm, PASTd algorithm, and Kalman-PASTd algorithm have high computational complexity. In the paper, we propose a computationally efficient DOA tracking algorithm in monostatic MIMO radar with automatic association.

In this paper, we reference the array-signal-processing DOA tracking idea in [31] to propose a DOA tracking 




FIgURE 1: The array structure of monostatic MIMO radar.

algorithm which is suitable for MIMO radar. Using the reduced-dimension transformation for the received signal of MIMO radar, we obtain the covariance matrix of reduceddimension transformation signal, and then we adopt an improved version for DOA tracking. The proposed algorithm can realize automatic association in DOA tracking. Error analysis and Cramér-Rao lower bound (CRLB) are also derived in this paper. Finally, the simulation results demonstrate the effectiveness and robustness of the proposed algorithm.

There are some differences between the work in [31] and the proposed algorithm. (1) Reference [31] proposed an effective DOA tracking algorithm in array signal processing, while we address DOA tracking problem for MIMO radar in the paper. (2) The DOA tracking algorithm in array signal processing in [31] requires the direction matrix of Vandermonde form. The direction matrix in MIMO radar is not a Vandermonde matrix, and the DOA tracking algorithm in [31] cannot be used directly for DOA tracking in MIMO radar. We employ the reduced-dimension transformation for the received signal to obtain the reduced-dimension direction matrix of Vandermonde form. (3) Our work improves the DOA tracking algorithm in [31] to enhance the DOA tracking performance in MIMO radar, since it fully uses the Toeplitz matrix property to eliminate the noise. Therefore, the proposed algorithm not only can be regarded as an extension of the work in [31], but also is an improved algorithm. Simulation results show that the proposed algorithm has much better DOA tracking performance than the DOA tracking algorithm in [31].

The reminder of this paper is structured as follows. Section 2 develops the data model for monostatic MIMO radar. Section 3 establishes our DOA tracking algorithm based on the elements of the covariance matrix of the reduced-dimension transformation signal. In Section 4, error analysis and Cramer-Rao lower bound (CRLB) are derived. In Section 5, simulation results are presented to verify the effectiveness of the proposed algorithm, while the conclusions are made in Section 6.

Notation. $(\cdot)^{T},(\cdot)^{H},(\cdot)^{-1}$, and $(\cdot)^{+}$denote transpose, conjugate-transpose, inverse, and pseudoinverse operations, respectively. $\operatorname{diag}(\mathbf{v})$ stands for diagonal matrix whose diagonal is a vector $\mathbf{v} ; \mathbf{I}_{K}$ is a $K \times K$ identity matrix; $\otimes$, o, and $\oplus$ are the Kronecker product, Khatri-Rao product, and Hadamard product, respectively. $E(\cdot)$ denotes the expectation operator. $\widehat{\mathbf{X}}$ denotes the estimated value of $\mathbf{X}$.

\section{Data Model}

We consider a monostatic MIMO radar system equipped with both uniform linear arrays (ULAs) for the transmit/receive array, in which $M$ elements and $N$ elements are arranged with half-wavelength spacing between adjacent antennas, respectively. The array structure of monostatic MIMO radar is shown in Figure 1. We assume that there are $K$ uncorrelated targets. At time $t$, the output of the matched filters at the receiver can be expressed as [9]

$$
\begin{aligned}
\mathbf{r}(t) & =\left[\mathbf{a}_{r}\left(\theta_{t, 1}\right) \otimes \mathbf{a}_{t}\left(\theta_{t, 1}\right), \ldots, \mathbf{a}_{r}\left(\theta_{t, K}\right) \otimes \mathbf{a}_{t}\left(\theta_{t, K}\right)\right] \mathbf{s}(t)+\mathbf{n}(t) \\
& =\mathbf{A}_{t} \mathbf{s}(t)+\mathbf{n}(t)
\end{aligned}
$$

where $\theta_{t, k}$ is the DOA of the $k$ th target at time $t . \mathbf{a}_{t}\left(\theta_{t, k}\right)=$ $\left[1, \exp \left(-j \pi \sin \theta_{t, k}\right), \ldots, \exp \left(-j(M-1) \pi \sin \theta_{t, k}\right)\right]^{T}, \mathbf{a}_{r}\left(\theta_{t, k}\right)=$ $\left[1, \exp \left(-j \pi \sin \theta_{t, k}\right), \ldots, \exp \left(-j(N-1) \pi \sin \theta_{t, k}\right)\right]^{T} \cdot \mathbf{s}(t)=$ $\left[s_{1}(t), s_{2}(t), \ldots, s_{K}(t)\right]^{T}$, and $s_{k}(t)=\rho_{k} e^{j 2 \pi f_{k} t}$ with $f_{k}$ being Doppler frequency and $\rho_{k}$ being the amplitude. We assume that $s_{i}(t)$ and $s_{j}(t)$ are uncorrelated for the different targets. $\mathbf{n}(t)$ is the received additive white Gaussian noise with noise vector of zeros mean and covariance matrix $\sigma^{2} \mathbf{I}_{M N}$ an $M N \times 1$ noise vector. $\otimes$ stands for Kronecker product. The matrix $\mathbf{A}_{t}$ is

$$
\mathbf{A}_{t}=\left[\mathbf{a}_{r}\left(\theta_{t, 1}\right) \otimes \mathbf{a}_{t}\left(\theta_{t, 1}\right), \ldots, \mathbf{a}_{r}\left(\theta_{t, K}\right) \otimes \mathbf{a}_{t}\left(\theta_{t, K}\right)\right] .
$$

During the interval $\left((t-1) T_{s}, t T_{s}\right], \theta_{t, k}$ is a constant and $J$ snapshots of sensor data are available for the signal processing.

\section{DOA Tracking Algorithm}

The DOA tracking algorithm of array signal processing in [31] requires the direction matrix of Vandermonde form. The direction matrix $\mathbf{A}_{t}$ in (2) is not a Vandermonde matrix, and the DOA tracking algorithm of array signal processing in [31] cannot be used directly for DOA tracking in MIMO radar. We employ the reduced-dimension transformation for the received signal to obtain the reduced-dimension direction matrix of Vandermonde form. In this paper, we reference the array-signal-processing DOA tracking idea in [31] to propose a low complexity DOA tracking algorithm which is suitable for MIMO radar.

3.1. Reduced-Dimension Transformation. $\mathbf{a}_{r}\left(\theta_{t, k}\right) \otimes \mathbf{a}_{t}\left(\theta_{t, k}\right)$ can be expressed by [9]

$$
\mathbf{a}_{r}\left(\theta_{t, k}\right) \otimes \mathbf{a}_{t}\left(\theta_{t, k}\right)=\mathbf{G b}\left(\theta_{t, k}\right),
$$


where $\mathbf{b}\left(\theta_{t, k}\right)=\left[1, \exp \left(-j \pi \sin \theta_{t, k}\right), \ldots, \exp (-j \pi(M+N-\right.$ 2) $\left.\left.\sin \theta_{t, k}\right)\right]^{T}$, and

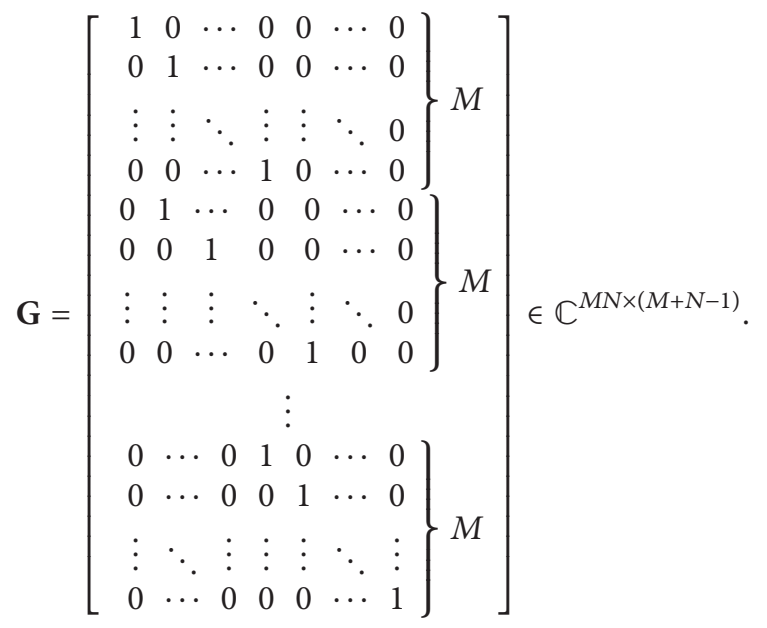

Then we define $\mathbf{W} \triangleq \mathbf{G}^{H} \mathbf{G}$ as follows:

$\mathbf{W}=\operatorname{diag}(1,2, \ldots, \underbrace{\min (M, N), \ldots, \min (M, N)}_{|M-N|+1}, \ldots, 2,1)$.

Using the reduced-dimension transformation $\mathbf{W}^{-1} \mathbf{G}^{H}$ for the receiver signal $\mathbf{r}(t)$, we obtain

$$
\begin{aligned}
\mathbf{y}(t) & =\mathbf{W}^{-1} \mathbf{G}^{H} \mathbf{r}(t) \\
& =\mathbf{W}^{-1} \mathbf{W}\left[\mathbf{b}\left(\theta_{t, 1}\right), \ldots, \mathbf{b}\left(\theta_{t, K}\right)\right] \mathbf{s}(t)+\mathbf{W}^{-1} \mathbf{G}^{H} \mathbf{n}(t) \\
& =\mathbf{B}_{t} \mathbf{s}(t)+\mathbf{W}^{-1} \mathbf{G}^{H} \mathbf{n}(t),
\end{aligned}
$$

where

$$
\mathbf{B}_{t}=\left[\mathbf{b}\left(\theta_{t, 1}\right), \ldots, \mathbf{b}\left(\theta_{t, K}\right)\right] \in \mathbb{C}^{(M+N-1) \times K} .
$$

$\mathbf{B}_{t} \in \mathbb{C}^{(M+N-1) \times K}$ is the direction matrix of Vandermonde form. Since the reduced-dimension matrix is sparse, its transformation adds less computational load.

The covariance matrix of $\mathbf{y}(t)$ in (6) is $\mathbf{R}_{t} \in$ $\mathbb{C}^{(M+N-1) \times(M+N-1)}$. Consider

$$
\begin{aligned}
\mathbf{R}_{t} & =E\left[\mathbf{y}(t) \mathbf{y}^{H}(t)\right] \\
& =\mathbf{B}_{t} \mathbf{R}_{s} \mathbf{B}_{t}{ }^{H}+\mathbf{R}_{n}^{\prime}(t),
\end{aligned}
$$

where $\mathbf{R}_{s}=E\left[\mathbf{s}(t) \mathbf{s}^{H}(t)\right]=\operatorname{diag}\left(\left|\rho_{1}\right|^{2}\left|\rho_{2}\right|^{2} \cdots\left|\rho_{K}\right|^{2}\right)$ and $\mathbf{R}_{n}^{\prime}(t)=\mathbf{W}^{-1} \mathbf{G}^{H} \mathbf{R}_{n} \mathbf{G} \mathbf{W}^{-1}$ with $\mathbf{R}_{n}=E\left[\mathbf{n}(t) \mathbf{n}(t)^{H}\right]$.

3.2. DOA Tracking. The direction matrix in the reduceddimension signal in (6) is a Vandermonde matrix, and we use the improved version of the DOA tracking in [31] for DOA tracking of MIMO radar.

We assume that $\theta_{t, k}$ is slowly varying. The DOA of the $K$ targets at time $t$ is $\theta_{t}=\left[\theta_{t, 1}, \theta_{t, 2}, \ldots, \theta_{t, K}\right]$. Similarly, the DOA at time $t+1$ is $\theta_{t+1}=\left[\theta_{t+1,1}, \theta_{t+1,2}, \ldots, \theta_{t+1, K}\right]$, with $\theta_{t+1, k}$ being the DOA of the $k$ th target at time $t+1$. We define

$$
\Delta \theta_{t}=\theta_{t+1}-\theta_{t},
$$

where $\Delta \theta_{t}=\left[\Delta \theta_{t, 1}, \Delta \theta_{t, 2}, \ldots, \Delta \theta_{t, K}\right]$ with $\Delta \theta_{t, k}=\theta_{t+1, k}-\theta_{t, k}$.

We define $\mathbf{R}_{t+1}$ as the covariance matrices of the signal $\mathbf{y}(t)$ at time $t+1$. The covariance matrix is

$$
\mathbf{R}_{t+1}=\mathbf{B}_{t+1} \mathbf{R}_{s} \mathbf{B}_{t+1}^{H}+\mathbf{R}_{n}^{\prime}(t+1),
$$

where $\mathbf{B}_{t+1}=\left[\mathbf{b}\left(\theta_{t+1,1}\right), \ldots, \mathbf{b}\left(\theta_{t+1, K}\right)\right]$ is the direction matrix at time $t+1$ and $\mathbf{b}\left(\theta_{t+1, k}\right)=\left[1, \exp \left(-j \pi \sin \theta_{t+1, k}\right), \ldots\right.$, $\left.\exp \left(-j \pi(M+N-2) \sin \theta_{t+1, k}\right)\right]^{T} . \mathbf{R}_{n}^{\prime}(t+1)$ is the covariance matrix of the noise.

Then we can obtain $[31,36]$

$$
\Delta \mathbf{R}_{t}=\mathbf{R}_{t+1}-\mathbf{R}_{t} .
$$

$\Delta \mathbf{R}_{t}$ is denoted by

$$
\Delta \mathbf{R}_{t}=\left(\mathbf{B}_{t+1} \mathbf{R}_{s} \mathbf{B}_{t+1}^{H}-\mathbf{B}_{t} \mathbf{R}_{s} \mathbf{B}_{t}^{H}\right)+\left(\mathbf{R}_{n}^{\prime}(t+1)-\mathbf{R}_{n}^{\prime}(t)\right) .
$$

We assume that the noise covariance matrix at time $t+1$ is approximately equal to that at time $t$, and then we have

$$
\Delta \mathbf{R}_{y} \simeq \mathbf{B}_{t+1} \mathbf{R}_{s} \mathbf{B}_{t+1}^{H}-\mathbf{B}_{t} \mathbf{R}_{s} \mathbf{B}_{t}^{H} .
$$

Using the Vandermonde characteristic of the matrices $\mathbf{B}_{t}$ and $\mathbf{B}_{t+1}$, the noiseless $\Delta \mathbf{R}_{t}$ in (13) can be denoted by [31, 32, 36]

$$
\Delta \mathbf{R}_{t}=\left[\begin{array}{ccccc}
0 & b_{1} & b_{2} & \cdots & b_{M+N-2} \\
b_{1}^{*} & 0 & b_{1} & \cdots & b_{M+N-3} \\
b_{2}^{*} & b_{1}^{*} & 0 & \cdots & b_{M+N-4} \\
\vdots & \vdots & \vdots & \ddots & \vdots \\
b_{M+N-2}^{*} & b_{M+N-3}^{*} & b_{M+N-4}^{*} & \cdots & 0
\end{array}\right],
$$

where

$$
\begin{array}{r}
b_{n}=\sum_{i=1}^{K} s_{i}\left(e^{j n \pi \sin \left(\theta_{t, i}+\Delta \theta_{t, i}\right)}-e^{j n \pi \sin \theta_{t, i}}\right), \\
n=1,2, \ldots, M+N-2,
\end{array}
$$

where $s_{i}$ is the $(i, i)$ element of the matrix $\mathbf{R}_{s}$ and $s_{i}=\left|\rho_{i}\right|^{2}$. $b_{n}$ in (15) can be expanded as $b_{n}=\sum_{i=1}^{K} s_{i}\left(e^{j n \pi\left[\sin \theta_{t, i} \cos \Delta \theta_{t, i}+\right.}\right.$ $\left.\left.\cos \theta_{t, i} \sin \Delta \theta_{t, i}\right]-e^{j n \pi \sin \theta_{t, i}}\right)$. Considering that $\Delta \theta_{t, i}$ is very small,

$$
\sin \left(\theta_{t, i}+\Delta \theta_{t, i}\right) \simeq \sin \theta_{t, i}+\Delta \theta_{t, i} \cos \theta_{t, i} .
$$


Then $b_{n}$ in (15) can be denoted by

$$
\begin{aligned}
b_{n} & \simeq \sum_{i=1}^{K} s_{i}\left(e^{j n \pi\left(\sin \theta_{t, i}+\Delta \theta_{t, i} \cos \theta_{t, i}\right)}-e^{j n \pi \sin \theta_{t, i}}\right) \\
& =\sum_{i=1}^{K} s_{i} e^{j \pi n \sin \theta_{t, i}}\left(e^{j n \pi \Delta \theta_{t, i} \cos \theta_{t, i}}-1\right) .
\end{aligned}
$$

Considering that $x$ is very small, $e^{x}-1 \simeq x$. And then $b_{n}$ in (17) can be rewritten as

$$
b_{n} \approx \sum_{i=1}^{K} s_{i} e^{n j \pi \sin \theta_{t, i}}\left(j n \pi \Delta \theta_{t, i} \cos \theta_{t, i}\right) .
$$

According to (18), we can construct the following matrix:

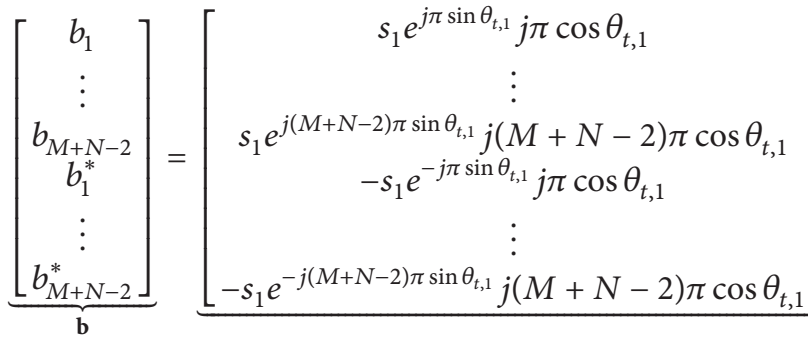



Equation (19) can be rewritten with matrix form

$$
\mathbf{V}_{t} \Delta \boldsymbol{\theta}_{t}=\mathbf{b}
$$

From (14), we find that $\Delta \mathbf{R}_{t}$ is a Toeplitz matrix, whose elements in a straight line paralleled to the principal diagonal are equal. We take the following processing to estimate $b_{n}$ and $b_{n}^{*}(n=1, \ldots, M+N-2)$ to eliminate the noise. Consider

$$
\begin{aligned}
& \widehat{b}_{n}=\frac{1}{M+N-1-n} \sum_{i=1}^{M+N-1-n} \Delta \mathbf{R}_{t}(i, n+i) \\
& \widehat{b}_{n}^{*}=\frac{1}{M+N-1-n} \sum_{i=1}^{M+N-1-n} \Delta \mathbf{R}_{t}(n+i, i),
\end{aligned}
$$

where $\Delta \mathbf{R}_{t}(i, j)$ is the $(i, j)$ element of the matrix $\Delta \mathbf{R}_{t}$. In the DOA tracking algorithm in [31], $\widehat{b}_{n}$ and $\widehat{b}_{n}^{*}$ are obtained through $\widehat{b}_{n}=\Delta \mathbf{R}_{t}(1, n+1)$ and $\widehat{b}_{n}^{*}=\Delta \mathbf{R}_{t}(n+1,1)$, respectively. The proposed algorithm fully uses the Toeplitz matrix property of $\Delta \mathbf{R}_{t}$ to eliminate the noise and improves the parameter estimation performance.

Using the method of least square, we get

$$
\Delta \widehat{\boldsymbol{\theta}}_{t}=\left(\mathbf{V}_{t}^{H} \mathbf{V}_{t}\right)^{-1} \mathbf{V}_{t}^{H} \widehat{\mathbf{b}}
$$

where $\widehat{\mathbf{b}}=\left[\begin{array}{llllll}\widehat{b}_{1} & \cdots & \widehat{b}_{M+N-2} & \widehat{b}_{1}^{*} & \cdots & \widehat{b}_{M+N-2}^{*}\end{array}\right]^{T}$.

Through the above analysis, the angles at time of $t$ and $t+1$ are automatically associated, and the proposed algorithm avoids an extra data association.

For the finite samples, the covariance matrix in (8) is estimated by $\widehat{\mathbf{R}}_{t}=\sum_{l=1}^{J} \mathbf{y}\left(t_{l}\right) \mathbf{y}^{H}\left(t_{l}\right)$, and we also get $\widehat{\mathbf{R}}_{t+1}$, which is the estimate of the covariance matrix at time $t+1$. We estimate $b_{n}$ and $\widehat{b}_{n}^{*}$ as follows:

$$
\begin{aligned}
& \widehat{b}_{n}=\frac{1}{M+N-1-n} \sum_{i=1}^{M+N-1-n} \Delta \widehat{\mathbf{R}}_{t}(i, n+i) \\
& \widehat{b}_{n}^{*}=\frac{1}{M+N-1-n} \sum_{i=1}^{M+N-1-n} \Delta \widehat{\mathbf{R}}_{t}(n+i, i),
\end{aligned}
$$

where $\Delta \widehat{\mathbf{R}}_{t}=\widehat{\mathbf{R}}_{t+1}-\widehat{\mathbf{R}}_{t}$. We use (22) to estimate $\Delta \boldsymbol{\theta}_{t}$, where $\widehat{\mathbf{b}}=\left[\begin{array}{llllll}\widehat{b}_{1} & \cdots & \widehat{b}_{M+N-2} & \widehat{b}_{1}^{*} & \cdots & \widehat{b}_{M+N-2}^{*}\end{array}\right]^{T}$, whose element is estimated via (23a) and (23b).

Till now, we show the major steps of DOA tracking algorithm for monostatic radar as follows.

Step 1. Use reduced-dimension matrix $\mathbf{W}^{-1} \mathbf{G}^{H}$ for the received signal $\mathbf{r}(t)$ and estimate the covariance matrix $\widehat{\mathbf{R}}_{t}$.

Step 2. Get covariance matrix $\widehat{\mathbf{R}}_{t+1}$ at time $t+1$, and we calculate $\Delta \widehat{\mathbf{R}}_{t}=\widehat{\mathbf{R}}_{t+1}-\widehat{\mathbf{R}}_{t}$.

Step 3. We compute $\widehat{b}_{n}$ and $\widehat{b}_{n}^{*}$ via (23a) and (23b) and construct $\widehat{\mathbf{b}}=\left[\begin{array}{llllll}\widehat{b}_{1} & \cdots & \widehat{b}_{M+N-2} & \widehat{b}_{1}^{*} & \cdots & \widehat{b}_{M+N-2}^{*}\end{array}\right]^{T}$.

Step 4. We estimate $\Delta \widehat{\boldsymbol{\theta}}_{t}$ via (22), and the DOA at time $t+1$ is $\widehat{\boldsymbol{\theta}}_{t+1}=\widehat{\boldsymbol{\theta}}_{t}+\Delta \widehat{\boldsymbol{\theta}}_{t}$.

Step 5. Repeat Steps 1-4 to estimate DOA at next time.

Remark 1. In this paper, the number of targets in MIMO radar is assumed to be preknown. If we have no knowledge about it, we may use the existing source-number estimation technique in [37] to obtain an estimate of the number of targets. 
TABLE 1: Complexity comparison.

\begin{tabular}{lc}
\hline Algorithm & Computational complexity \\
\hline PAST [25] & $O\left(\left(3 M N K+K^{2}\right) J+3 K^{2} M(N-1)+K^{3}\right)$ \\
PASTd [26] & $O\left((4 M N K+K) J+3 K^{2} M(N-1)+K^{3}\right)$ \\
The proposed algorithm & $O\left((M+N-1)^{2} J+2 K^{2}(M+N-2)+\right.$ \\
& $\left.K^{3}+2 K(M+N-2)+K^{2}\right)$ \\
\hline
\end{tabular}

Remark 2. The initial angles in DOA tracking are obtained by ESPRIT algorithm or other DOA algorithms. The initial DOA is $\widehat{\boldsymbol{\theta}}_{1}$, and we get angles $\widehat{\boldsymbol{\theta}}_{m}=\widehat{\boldsymbol{\theta}}_{1}+\sum_{i=1}^{m-1} \Delta \widehat{\boldsymbol{\theta}}_{i}$.

Remark 3. We assume that the noise covariance matrices of adjacent time are approximately equal. The noise component in (12) will be eliminated no matter which type of noise. The proposed algorithm still works well for the case of the colored noise.

Remark 4. The proposed algorithm works well in condition of small value of $\Delta \theta_{t, k}$. When $\Delta \theta_{t, k}$ becomes large, the proposed algorithm may fail to work.

\subsection{Complexity Analysis and Advantages of the Proposed} Algorithm. Since the reduced-dimension matrix is sparse, its transformation adds less computational load. The proposed algorithm does not require eigenvalue decomposition of the covariance matrix and avoids an extra data association. For the proposed algorithm, the calculation of the covariance matrix requires $O\left((M+N-1)^{2} J\right)$, and the computation of $\left(\mathbf{V}_{t}^{H} \mathbf{V}_{t}\right)^{-1} \mathbf{V}_{t}^{H} \widehat{\mathbf{b}}$ needs $\left(2 K^{2}(M+N-2)+K^{3}+2 K(M+N-\right.$ $\left.2)+K^{2}\right)$. The major computational complexity of the proposed algorithm is $O\left((M+N-1)^{2} J+2 K^{2}(M+N-2)+K^{3}+2 K(M+\right.$ $N-2)+K^{2}$ ). Table 1 and Figure 2 show the computational complexity comparison among the proposed algorithm and other DOA tracking algorithms. We find that the proposed algorithm has much lower computational load than PAST in [25] and PASTd in [26].

The advantages of the proposed algorithm can be presented as follows.

(1) The proposed algorithm does not require eigenvalue decomposition of the covariance matrix and has lower complexity than the conventional DOA tracking algorithms including PAST and PASTd.

(2) The proposed algorithm can implement automatic association of DOA for monostatic MIMO radar.

(3) The proposed algorithm has much better DOA tracking performance than the DOA tracking algorithm in [31], which will be shown in Section 5.

\section{Error Analysis and CRLB}

In this section, we derive the variance of DOA tracking and CRLB. We assume that the observation noise variances are almost the same at adjacent time. When computing $\Delta \boldsymbol{\theta}$, we use some approximate calculations such as $e^{x}-1 \approx x$ and

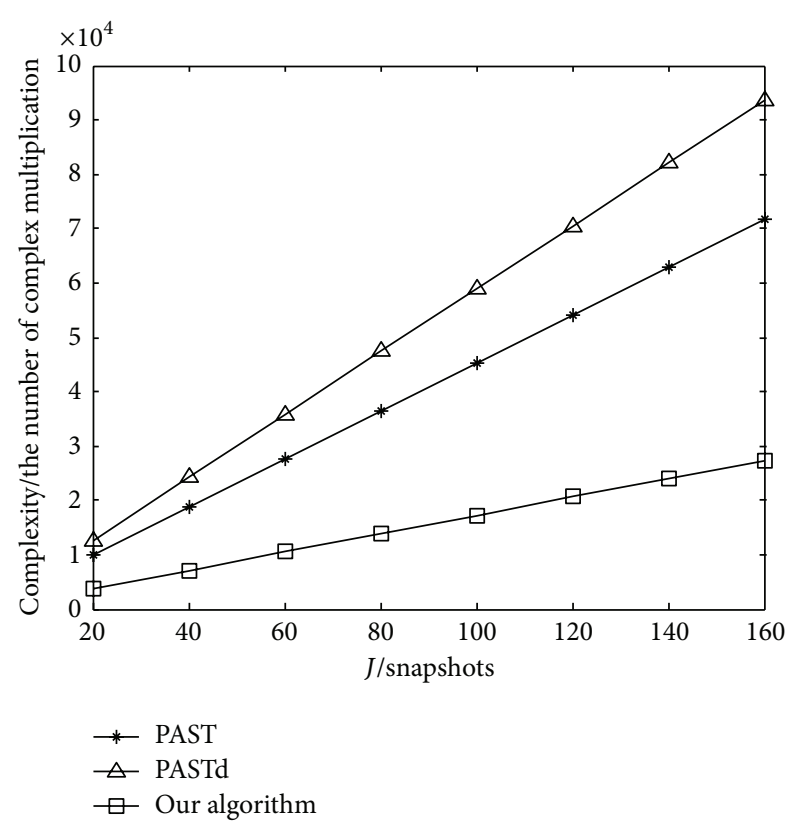

FIgURE 2: Complexity comparison with $M=8, N=6, K=3$, and different $J$.

$\sin x \approx x$ when $x$ is smaller. This leads to a little difference compared with the perfect value. Consider

$$
\begin{gathered}
\sin x=x-\frac{x^{3}}{3 !}+\Lambda \\
\cos x=1-\frac{x^{2}}{2 !}+\Lambda^{\prime} \\
e^{x}-1=x+\Lambda^{\prime \prime},
\end{gathered}
$$

where $\Lambda, \Lambda^{\prime}, \Lambda^{\prime \prime}$ are the high-order expansion terms.

According to (16), (17), (18), (24a), (24b), and (24c), we have

$$
\begin{aligned}
& b_{n}= \sum_{i=1}^{K} s_{i}\left(e^{j n \pi\left[\sin \theta_{t, i} \cos \Delta \theta_{t, i}+\cos \theta_{t, i} \sin \Delta \theta_{t, i}\right]}-e^{j n \pi \sin \theta_{t, i}}\right) \\
&= \sum_{i=1}^{K} s_{i}\left(e^{j n \pi\left[\sin \theta_{t, i}\left(1-\left(\Delta \theta_{t, i}^{2} / 2 !\right)+\Lambda^{\prime}\right)+\cos \theta_{t, i}\left(\Delta \theta_{t, i}-\left(\Delta \theta_{t, i}^{3} / 3 !\right)+\Lambda\right)\right]}\right. \\
&\left.-e^{j n \pi \sin \theta_{t, i}}\right) \\
&= \sum_{i=1}^{K} s_{i}\left(e^{j n \pi\left[\sin \theta_{t, i}+\cos \theta_{t, i} \Delta \theta_{t, i}\right]}\right. \\
& \quad \times e^{j n \pi\left[\sin \theta_{t, i}\left(-\left(\Delta \theta_{t, i}^{2} / 2 !\right)+\Lambda^{\prime}\right)+\cos \theta_{t, i}\left(-\left(\Delta \theta_{t, i}^{3} / 3 !\right)+\Lambda\right)\right]} \\
&=\sum_{i=1}^{K} s_{i} e^{j n \pi \sin \theta_{t, i}} \\
& \quad \times\left(e^{j n \pi \sin \theta_{t, i}}\right)
\end{aligned}
$$


$\left.\times e^{j n \pi\left[\sin \theta_{t, i}\left(-\left(\Delta \theta_{t, i}^{2} / 2 !\right)+\Lambda^{\prime}\right)+\cos \theta_{t, i}\left(-\left(\Delta \theta_{t, i}^{3} / 3 !\right)+\Lambda\right)\right]}-1\right)$

$$
\begin{gathered}
=\sum_{i=1}^{K} s_{i} e^{j n \pi \sin \theta_{t, i}} \\
\times\left(j n \pi \cos \theta_{t, i} \Delta \theta_{t, i}\right. \\
+j n \pi\left[\sin \theta_{t, i}\left(-\frac{\Delta \theta_{t, i}^{2}}{2 !}+\Lambda^{\prime}\right)\right. \\
\left.+\cos \theta_{t, i}\left(-\frac{\Delta \theta_{t, i}^{3}}{3 !}+\Lambda\right)+j \Lambda^{\prime \prime}\right] \\
\left.+j \Lambda^{\prime \prime}\right) \quad \\
=\sum_{i=1}^{K} s_{i} e^{j n \pi \sin \theta_{t, i} j n \pi \cos \theta_{t, i} \Delta \theta_{t, i}} \\
+\sum_{i=1}^{K} s_{i} e^{j n \pi \sin \theta_{t, i}(j n \pi}\left[\sin \theta_{t, i}\left(-\frac{\Delta \theta_{t, i}^{2}}{2 !}+\Lambda^{\prime}\right)\right. \\
\left.+\cos \theta_{t, i}\left(-\frac{\Delta \theta_{t, i}^{3}}{3 !}+\Lambda\right)\right] \\
\left.+j \Lambda^{\prime \prime}\right) .
\end{gathered}
$$

$\partial b_{n}$ is estimation error of $b_{n}$, and $\partial b_{n}$ can be shown as follows:

$$
\begin{aligned}
\partial b_{n}=\sum_{i=1}^{K} s_{i} e^{j n \pi \sin \theta_{t, i}}(j n \pi & {\left[\sin \theta_{t, i}\left(-\frac{\Delta \theta_{t, i}^{2}}{2 !}+\Lambda^{\prime}\right)\right.} \\
& \left.\left.+\cos \theta_{t, i}\left(-\frac{\Delta \theta_{t, i}^{3}}{3 !}+\Lambda\right)\right]+j \Lambda^{\prime \prime}\right) .
\end{aligned}
$$

According to (26) and (22), the variance of $\Delta b_{n}$ is denoted by

$$
\begin{aligned}
E\left[\left|\partial b_{n}\right|^{2}\right]= & \frac{1}{M+N-1-n} \\
& \times \sum_{i=1}^{K} s_{i}^{2}\left[n \pi \sin \theta_{t, i}\left(-\frac{\Delta \theta_{t, i}^{2}}{2 !}+\Lambda^{\prime}\right)\right. \\
& \left.\quad+n \pi \cos \theta_{t, i}\left(-\frac{\Delta \theta_{t, i}^{3}}{3 !}+\Lambda\right)+\Lambda^{\prime \prime}\right]^{2}
\end{aligned}
$$

And we have

$$
\begin{gathered}
E\left[\partial b_{i} \partial b_{j}^{*}\right]=0, \quad \forall i \neq j \\
E\left[\partial b_{n}^{2}\right]=0 .
\end{gathered}
$$

We define that $\partial \mathbf{b}$ is the estimation error of $\mathbf{b}$. $\partial \mathbf{b}=$ $\left[\partial b_{1}, \ldots, \partial b_{M+N-2}, \partial b_{1}^{*}, \ldots, \partial b_{M+N-2}^{*}\right]^{T}$. Then the variance of $\partial \mathbf{b}$ is

$$
\left.\left.E\left[|\partial \mathbf{b}|^{2}\right]=\left[\begin{array}{c}
E\left[\left|\partial b_{1}\right|^{2}\right] \\
\vdots \\
E\left[\left|\partial b_{M+N-2}\right|^{2}\right] \\
E\left[\left|\partial b_{1}\right|^{2}\right] \\
\vdots \\
E\left[\left|\partial b_{M+N-2}\right|^{2}\right]
\end{array}\right]=\left[\begin{array}{c}
\frac{1}{M+N-2} \sum_{i=1}^{K} s_{i}^{2}\left[\pi \sin \theta_{t, i}\left(-\frac{\Delta \theta_{t, i}^{2}}{2 !}+\Lambda^{\prime}\right)+\pi \cos \theta_{t, i}\left(-\frac{\Delta \theta_{t, i}^{3}}{3 !}+\Lambda\right)+\Lambda^{\prime \prime}\right]^{2} \\
\vdots \\
\frac{1}{M+N-2} \sum_{i=1}^{K} s_{i}^{2}\left[\pi \sin \theta_{t, i}\left(-\frac{\Delta \theta_{t, i}^{2}}{2 !}+\Lambda^{\prime}\right)+\pi \cos \theta_{t, i}\left(-\frac{\Delta \theta_{t, i}^{3}}{3 !}+\Lambda\right)+\Lambda^{\prime \prime}\right]^{2} \\
\vdots \\
\sum_{i=1}^{K} s_{i}^{2}\left[(M+N-2) \pi \sin \theta_{t, i}\left(-\frac{\Delta \theta_{t, i}^{2}}{2 !}+\Lambda^{\prime}\right)+(M+N-2) \pi \cos \theta_{t, i}\left(-\frac{\Delta \theta_{t, i}^{2}}{3 !}+\Lambda\right)+\Lambda_{t, i}^{3}\right. \\
3 !
\end{array}\right]^{\prime \prime}+\Lambda^{\prime \prime}\right]^{2}\right]^{2}
$$

According to [38], we get the variance of $\Delta \theta_{t, i}$

$$
\operatorname{var}\left[\Delta \theta_{t, i}\right]=\frac{E\left[\left|\mathbf{V}_{t, i}^{+} \partial \mathbf{b}\right|^{2}\right]+\operatorname{Re}\left(E\left[\mathbf{V}_{t, i}^{+} \partial \mathbf{b}\right]^{2}\right)}{2}
$$

where $\mathbf{V}_{t, i}^{+}$denotes the $i$ th row of $\mathbf{V}_{t}^{+}$and $\mathbf{V}_{t}^{+}$is the pseudoinverse of $\mathbf{V}_{t}$. According to (27a), (27b), and (27c), we get

$$
\begin{aligned}
& \operatorname{var}\left[\Delta \theta_{t, i}\right] \\
& =\frac{\mathbf{V}_{t, i}^{+} \operatorname{diag}\left(E\left[|\partial \mathbf{b}|^{2}\right]\right) \mathbf{V}_{t, i}^{+H}+\operatorname{Re}\left(\mathbf{V}_{t, i}^{+} E\left[\partial \mathbf{b} \partial \mathbf{b}^{T}\right] \mathbf{V}_{t, i}^{+T}\right)}{2}
\end{aligned}
$$




$$
=\frac{\mathbf{V}_{t, i}^{+} \operatorname{diag}\left(E\left[|\partial \mathbf{b}|^{2}\right]\right) \mathbf{V}_{t, i}^{+H}}{2}
$$

where $E\left[|\partial \mathbf{b}|^{2}\right]$ is shown in (28).

According to [39], we derive the CRLB for DOA estimation in monostatic MIMO-radar at time $t$. Consider

$$
\operatorname{CRLB}(t)=\frac{\sigma^{2}}{2 J}\left\{\operatorname{Re}\left[\left(\mathbf{D}^{H} \Pi_{\mathbf{A}_{t}}^{\perp} \mathbf{D}\right) \oplus \mathbf{P}^{T}\right]\right\}^{-1},
$$

where $\Pi_{\mathbf{A}_{t}}^{\perp}=\mathbf{I}_{M N}-\mathbf{A}_{t}\left(\mathbf{A}_{t}^{H} \mathbf{A}_{t}\right)^{-1} \mathbf{A}_{t}^{H}, \mathbf{D}=\left[\mathbf{d}_{1}, \mathbf{d}_{2}, \ldots, \mathbf{d}_{K}\right]$, $\mathbf{d}_{k}=\partial\left(\mathbf{a}_{r}\left(\theta_{t, k}\right) \otimes \mathbf{a}_{t}\left(\theta_{t, k}\right) / \partial \theta_{t, k}\right.$, and

$\mathbf{P}=(1 / J) \sum_{n=1}^{J} \mathbf{s}\left(t_{n}\right) \mathbf{s}^{H}\left(t_{n}\right) . \oplus$ stands for Hadamard product.

Then we can define the average CRLB as follows:

$$
\operatorname{CRLB}=\frac{1}{T} \sum_{t=1}^{T} \operatorname{CRLB}(t),
$$

where $T$ is the total tracking time.

\section{Simulation Results}

The Monte Carlo simulations are adopted to assess DOA tracking performance of the proposed algorithm. We suppose that there are three moving targets. We define root-mean square error (RMSE) as

$$
\text { RMSE }=\frac{1}{K} \sum_{k=1}^{K} \sqrt{\frac{1}{F} \sum_{f=1}^{F}\left[\frac{1}{T} \sum_{t=1}^{T}\left(\widehat{\theta}_{k, f, t}-\theta_{k, f, t}\right)^{2}\right]},
$$

where $\hat{\theta}_{k, f, t}$ is the estimate of DOA $\theta_{t, k}$ of the $f$ th Monte Carlo trial. $F$ is the times of Monte Carlo trial and $F=$ 1000 . We note that $M, N$, and $K$ are the number of transmit antennas, receive antennas, and targets, respectively. During the tracking procedure, the targets are tracked over an interval of $100 \mathrm{~s}$ with $T_{s}=1 \mathrm{~s}$. During each $1 \mathrm{~s}$ interval, $J$ snapshots of sensor data are generated and used to estimate DOA.

Figure 3 depicts the DOA tracking result of the proposed algorithm for $M=8, N=6, K=3, J=100$, and SNR = $15 \mathrm{~dB}$. It is shown that the proposed algorithm can track the DOA in monostatic MIMO radar effectively.

Figures 4-5 show the DOA tracking result of the proposed algorithm for other moving trajectories of targets with $M=8$, $N=6, K=3, J=100$, and SNR $=15 \mathrm{~dB}$. From Figures 45 we find that the proposed algorithm can work well, which proves the robustness of the proposed algorithm.

Figure 6 shows the DOA tracking performance comparison with $M=8, N=6$, and $K=3$, where we compare the proposed algorithm against the DOA tracking algorithm in [31], Kalman-PASTd algorithm in [35], and CRLB. It is shown in Figure 6 that the proposed algorithm has better DOA tracking performance than the DOA tracking algorithm in [31], since the proposed algorithm fully uses the Toeplitz matrix property of $\Delta \mathbf{R}_{t}$ to eliminate the noise and improves

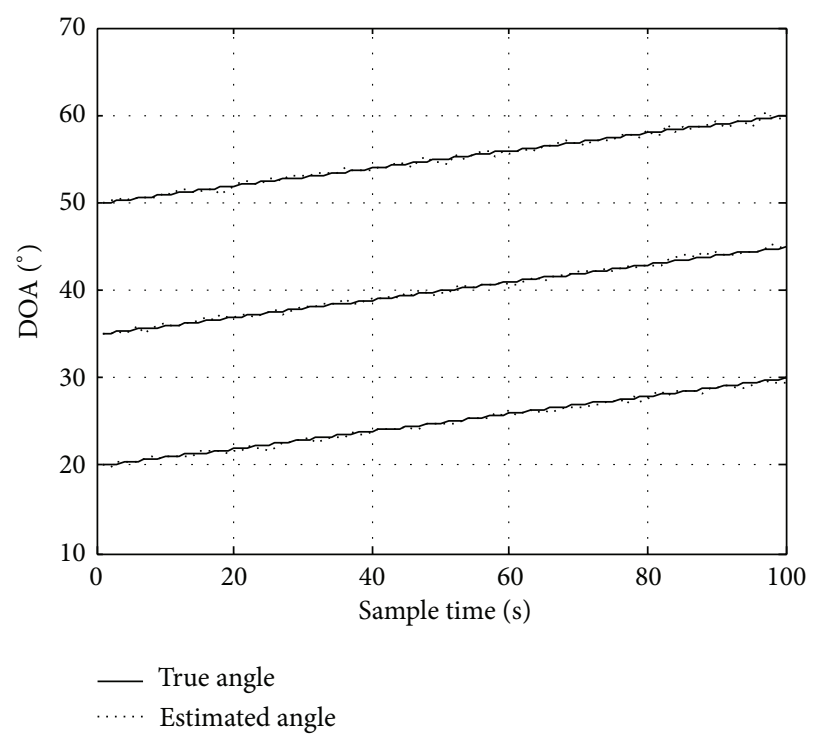

FIGURE 3: DOA tracking results of the proposed algorithm at $\mathrm{SNR}=$ $15 \mathrm{~dB}$ (Scene 1).

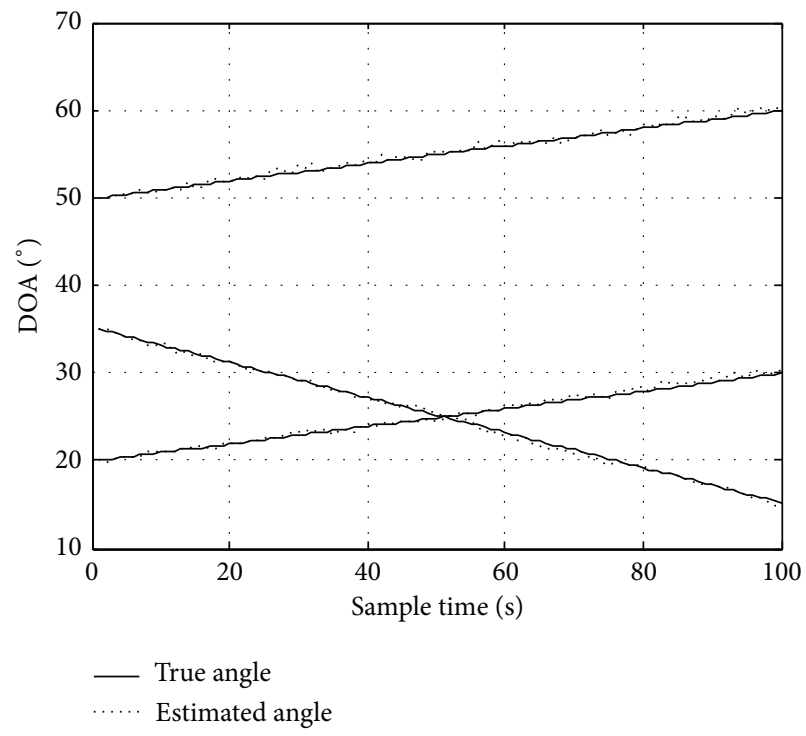

FIGURE 4: DOA tracking results of the proposed algorithm at SNR $=15 \mathrm{~dB}$ (Scene 2$)$.

the estimation performance. The proposed algorithm has less DOA tracking performance than Kalman-PASTd algorithm, which has a heavy computational complexity.

Figure 7 investigates the DOA tracking performance of the proposed algorithm with different values of $K$. From Figure 7 we find that DOA tracking performance of the proposed algorithm improves with the decreasing of $K$. When $K$ increases, the interference will enhance.

Figures 8-9 show DOA tracking performance with different $M / N$. It is clear that the DOA tracking performance of the proposed algorithm is improved as the number of trans$\mathrm{mit} /$ receive antennas increases. Multiple transmit/receive 


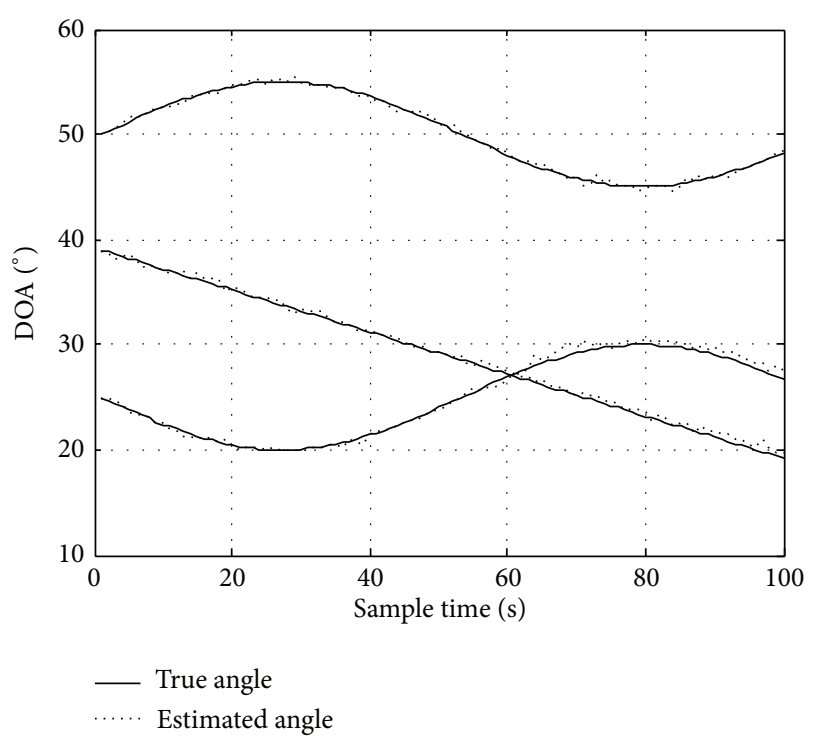

FIGURE 5: DOA tracking results of the proposed algorithm at SNR = $15 \mathrm{~dB}$ (Scene 3).

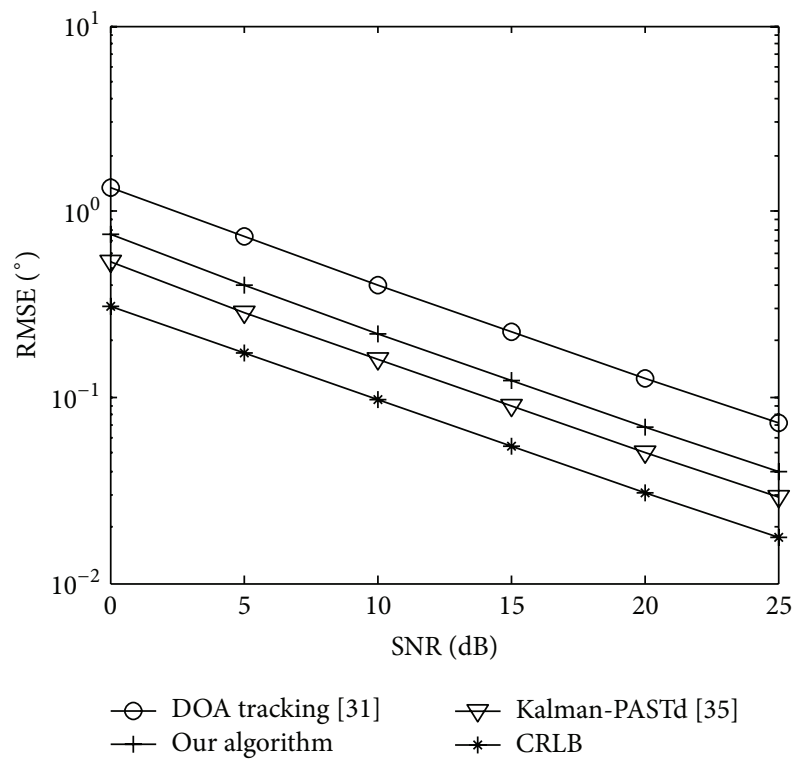

FIGURE 6: DOA tracking performance comparison with $M=8$ and $N=6$.

antennas improve DOA tracking performance because of diversity gain.

Figure 10 depicts the DOA tracking performance of the proposed algorithm with $M=8, N=6, K=3, J=100$, and SNR $=15 \mathrm{~dB}$, and the different DOA spacing between two consecutive observations is considered. It is shown in Figure 10 that the angle tracking performance of the proposed algorithm degrades with increasing of DOA spacing between two consecutive observations.

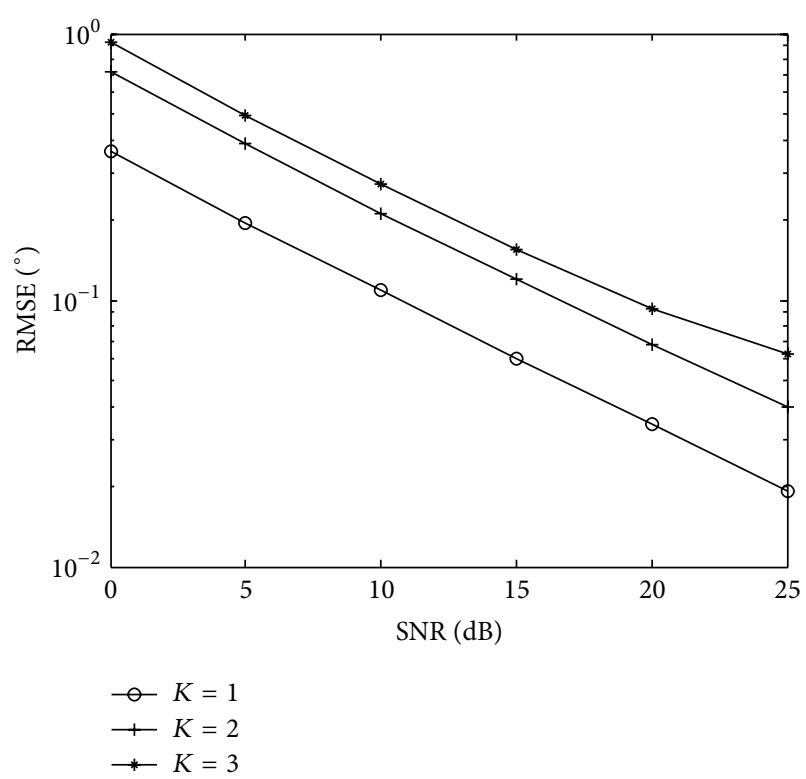

FIGURE 7: DOA tracking with different values of $K$.

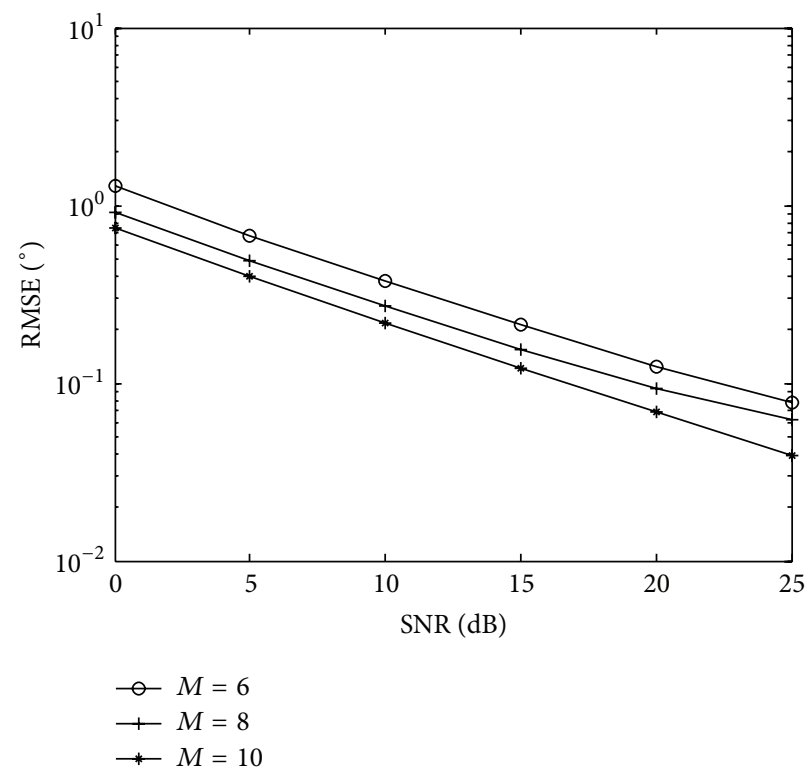

Figure 8: DOA tracking with different values of $M$.

\section{Conclusions}

In this paper we have presented the DOA tracking of multiple moving targets for monostatic MIMO radar. The proposed algorithm obtains DOA estimation of the target via the difference in previous and current covariance matrix of the reduced-dimension transformation signals, and the proposed algorithm reduces the computational complexity and realizes automatic association of DOA. Error analysis and CRLB of DOA tracking are derived in the paper. The simulation results demonstrate effectiveness and robustness of the proposed algorithm. Our research provides technical support for the practical application of MIMO radar. 


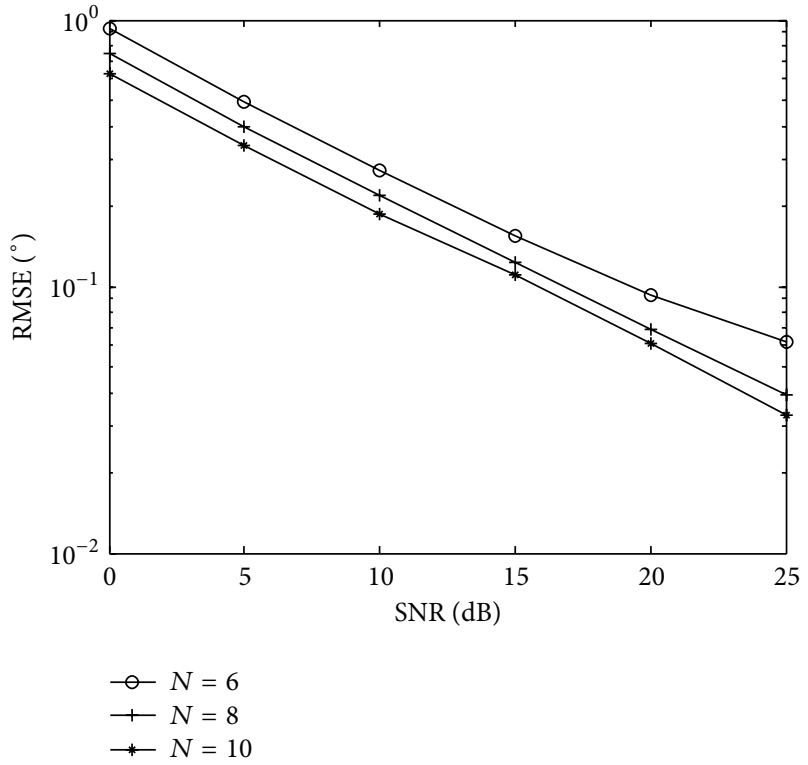

Figure 9: DOA tracking with different values of $N$.

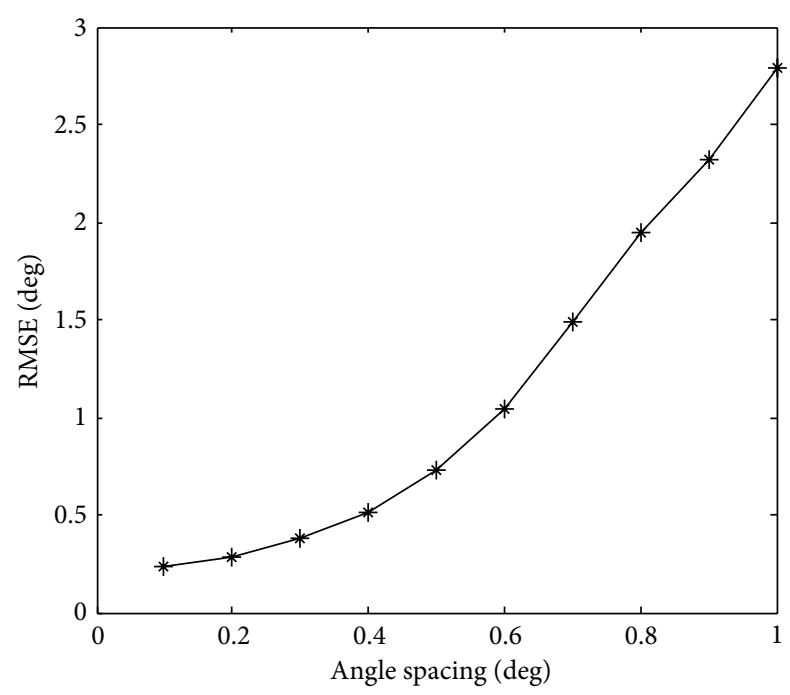

FIGURE 10: DOA tracking performance with different angle spacing.

\section{Conflict of Interests}

The authors declare that there is no conflict of interests regarding the publication of this paper.

\section{Acknowledgments}

This work is supported by China NSF Grants (61371169, 61301108, and 61071164), Jiangsu Planned Projects for Postdoctoral Research Funds (1201039C), China Postdoctoral Science Foundation (2012M521099 and 2013M541661), Open Project of Key Laboratory of Modern Acoustic of Ministry of Education (Nanjing University), Aeronautical Science Foundation of China (20120152001), Qing Lan Project, Priority Academic Program Development of Jiangsu High Education
Institutions, and Fundamental Research Funds for the Central Universities (NS2013024, kfji130114, and kfjj130115).

\section{References}

[1] E. Fishler, A. Haimovich, R. Blum, D. Chizhik, L. Cimini, and R. Valenzuela, "MIMO radar: an idea whose time has come," in Proceedings of the IEEE Radar Conference, pp. 71-78, April 2004.

[2] X. Li, Z. Zhang, W. Mao, X. Wang, J. Lu, and W. Wang, "A study of frequency diversity MIMO radar beamforming," in Proceedings of the IEEE 10th International Conference on Signal Processing (ICSP '10), pp. 352-356, Beijing, China, October 2010.

[3] J. Li and P. Stoica, "MIMO radar-diversity means superiority," in Proceedings of 14th Annual Workshop Adaptive Sensor Array Processing, Lexington, Mass, USA, June 2006.

[4] J. Li and P. Stoica, "MIMO radar with colocated antennas," IEEE Signal Processing Magazine, vol. 24, no. 5, pp. 106-114, 2007.

[5] A. M. Haimovich, R. S. Blum, and L. J. Cimini, "MIMO radar with widely separated antennas," IEEE Signal Processing Magazine, vol. 25, no. 1, pp. 116-129, 2008.

[6] C. Jinli, G. Hong, and S. Weimin, "Angle estimation using ESPRIT without pairing in MIMO radar," Electronics Letters, vol. 44, no. 24, pp. 1422-1423, 2008.

[7] J. Li and X. Zhang, "Performance analysis of ESPRIT for angle estimation in bistatic MIMO radar," in Proceedings of the International Conference on Computer, Electrical, and Systems Sciences (CESSE '11), pp. 400-403, Wuhan, China, 2011.

[8] M. L. Bencheikh and Y. Wang, "Joint DOD-DOA estimation using combined ESPRIT-MUSIC approach in MIMO radar," Electronics Letters, vol. 46, no. 15, pp. 1081-1083, 2010.

[9] M. L. Bencheikh, Y. Wang, and H. He, "Polynomial root finding technique for joint DOA DOD estimation in bistatic MIMO radar," Signal Processing, vol. 90, no. 9, pp. 2723-2730, 2010.

[10] X. Zhang and D. Xu, "Angle estimation in MIMO radar using reduced-dimension Capon," Electronics Letters, vol. 46, no. 12, pp. 860-861, 2010.

[11] X. Zhang, Y. Huang, C. Chen, J. Li, and D. Xu, "Reducedcomplexity Capon for direction of arrival estimation in a monostatic multiple-input multiple-output radar," IET Radar, Sonar and Navigation, vol. 6, no. 8, pp. 796-801, 2012.

[12] X. Gao, X. Zhang, G. Feng, Z. Wang, and D. Xu, "On the MUSIC-derived approaches of angle estimation for bistatic MIMO radar," in Proceedings of the International Conference on Wireless Networks and Information Systems (WNIS '09), pp. 343-346, Shanghai, China, December 2009.

[13] J. Li and X. Zhang, "Improved Joint DOD and DOA estimation for mimo array with velocity receive sensors," IEEE Signal Processing Letters, vol. 18, no. 12, pp. 717-720, 2011.

[14] X. Zhang, L. Xu, and D. Xu, "Direction of departure (DOD) and direction of arrival (DOA) estimation in MIMO radar with reduced-dimension MUSIC," IEEE Communications Letters, vol. 14, no. 12, pp. 1161-1163, 2010.

[15] F. Liu and J. Wang, "AD-MUSIC for jointly DOA and DOD estimation in bistatic MIMO radar system," in Proceedings of the International Conference on Computer Design and Applications (ICCDA '10), pp. 455-458, Qinhuangdao, China, June 2010.

[16] X. Zhang, Z. Xu, L. Xu, and D. Xu, "Trilinear decompositionbased transmit angle and receive angle estimation for multipleinput multiple-output radar," IET Radar, Sonar \& Navigation, vol. 5, no. 6, pp. 626-631, 2011. 
[17] H. Wu and X. Zhang, "DOA tracking in monostatic MIMO radar using PARAFAC-RLST algorithm," in Proceedings of the 3rd International Conference on Information and Systems Engineering (ICISE '11), pp. 958-961, Amsterdam, The Netherlands, July 2011.

[18] D. Nion and N. D. Sidiropoulos, "Adaptive algorithms to track the PARAFAC decomposition of a third-order tensor," IEEE Transactions on Signal Processing, vol. 57, no. 6, pp. 2299-2310, 2009.

[19] X. Zhang, H. Wu, J. Li, and D. Xu, "Computationally efficient DOD and DOA estimation for bistatic MIMO radar with propagator method," International Journal of Electronics, vol. 99, no. 9, pp. 1207-1221, 2012.

[20] Z. D. Zheng and J. Y. Zhang, "Fast method for multi-target localisation in bistatic MIMO radar," Electronics Letters, vol. 47, no. 2, pp. 138-139, 2011.

[21] J. Li and X. Zhang, "2D-angle estimation algorithm using quaternion theory in bistatic MIMO-radar," in Proceedings of the 3rd International Conference on Information Science and Engineering (ICISE '11), pp. 752-755, 2011.

[22] J. Li, X. Zhang, and F. Wang, "Quaternion root-MUSIC algorithm for angle estimation in bistatic MIMO radar," Journal of Electronics and Information Technology, vol. 34, no. 2, pp. 300304, 2012.

[23] Y. Yu, A. P. Petropulu, and H. V. Poor, "MIMO radar using compressive sampling," IEEE Journal on Selected Topics in Signal Processing, vol. 4, no. 1, pp. 146-163, 2010.

[24] Y. Liu, M. Y. Wu, and S. J. Wu, "Fast OMP algorithm for 2D angle estimation in MIMO radar," Electronics Letters, vol. 46, no. 6, pp. 444-445, 2010.

[25] B. Yang, "Projection approximation subspace tracking," IEEE Transactions on Signal Processing, vol. 43, no. 1, pp. 95-107, 1995.

[26] B. Yang, "Extension of the PASTd algorithm to both rank and subspace tracking," IEEE Signal Processing Letters, vol. 2, no. 9, pp. 179-182, 1995.

[27] S. Ouyang and Y. Hua, "Bi-iterative least-square method for subspace tracking," IEEE Transactions on Signal Processing, vol. 53, no. 8, part 2, pp. 2984-2996, 2005.

[28] P. Strobach, "Bi-iteration SVD subspace tracking algorithms," IEEE Transactions on Signal Processing, vol. 45, no. 5, pp. 12221240, 1997.

[29] S. B. Park, C. S. Ryu, and K. K. Lee, "Multiple target angle tracking algorithm using predicted angles," IEEE Transactions on Aerospace and Electronic Systems, vol. 30, no. 2, pp. 643-648, 1994.

[30] C. K. Sword, M. Simaan, and E. W. Kamen, "Multiple target angle tracking using sensor array outputs," IEEE Transactions on Aerospace and Electronic Systems, vol. 26, no. 2, pp. 367-373, 1990.

[31] H. Zhang, L. Zhang, S. Wu, and Y. Liu, "A new multiple targets angle tracking method," Journal of Electronics and Information Technology, vol. 29, no. 12, pp. 2840-2842, 2007.

[32] H. Zhang, S. Wu, L. Zhang, and Y. Liu, "Multiple targets angle tracking algorithm based on the elements of the covariance matrix," Journal of Xidian University, vol. 35, no. 5, pp. 785-792, 2008.

[33] C. R. Rao, L. Zhang, and L. C. Zhao, "Multitarget angle tracking an algorithm for data association," IEEE Transactions on Signal Processing, vol. 42, no. 2, pp. 459-462, 1994.

[34] H. Wu and X. Zhang, "DOD and DOA tracking algorithm for bistatic MIMO radar using PASTD without additional angles pairing," in Proceedings of the IEEE 5th International Conference on Advanced Computational Intelligence (ICACI '12), pp. 11321136, October 2012.

[35] X. F. Zhang, J. F. Li, G. P. Feng, and H. L. Wu, "Kalman-PASTd based DOA tracking algorithm for monostatic MIMO radar," in Proceedings of the International Conference on Information, Services and Management Engineering (ISME '11), pp. 220-224, 2011.

[36] W. Hailang, Multi-target angle estimation and tracking in monostatic MIMO radar system [M.S. thesis], Nanjing University of Aeronautics and Astronautics, 2013.

[37] J. Xin, N. Zheng, and A. Sano, "Simple and efficient nonparametric method for estimating the number of signals without eigendecomposition," IEEE Transactions on Signal Processing, vol. 55, no. 4, pp. 1405-1420, 2007.

[38] B. D. Rao and K. V. S. Hari, "Performance analysis of ESPRIT and TAM in determining the direction of arrival of plane waves in noise," IEEE Transactions on Speech and Signal Processing, vol. 37, no. 12, pp. 1990-1995, 1989.

[39] P. Stoica and A. Nehorai, "Performance study of conditional and unconditional direction-of-arrival estimation," IEEE Transactions on Acoustics, Speech, and Signal Processing, vol. 38, no. 10, pp. 1783-1795, 1990. 

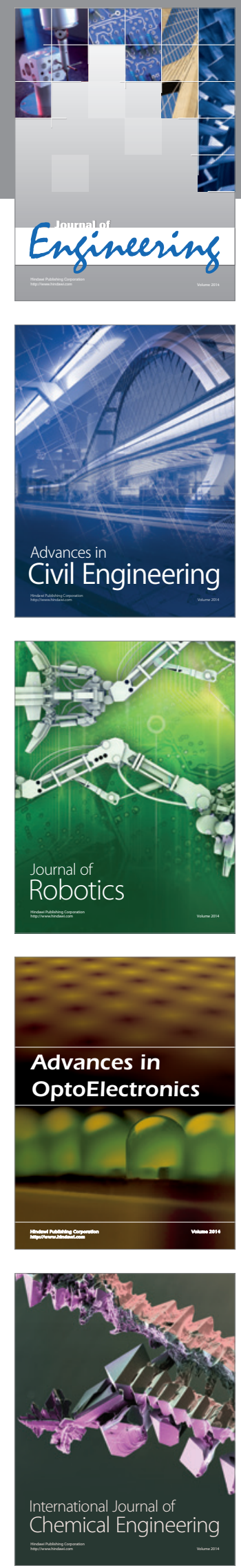



The Scientific World Journal
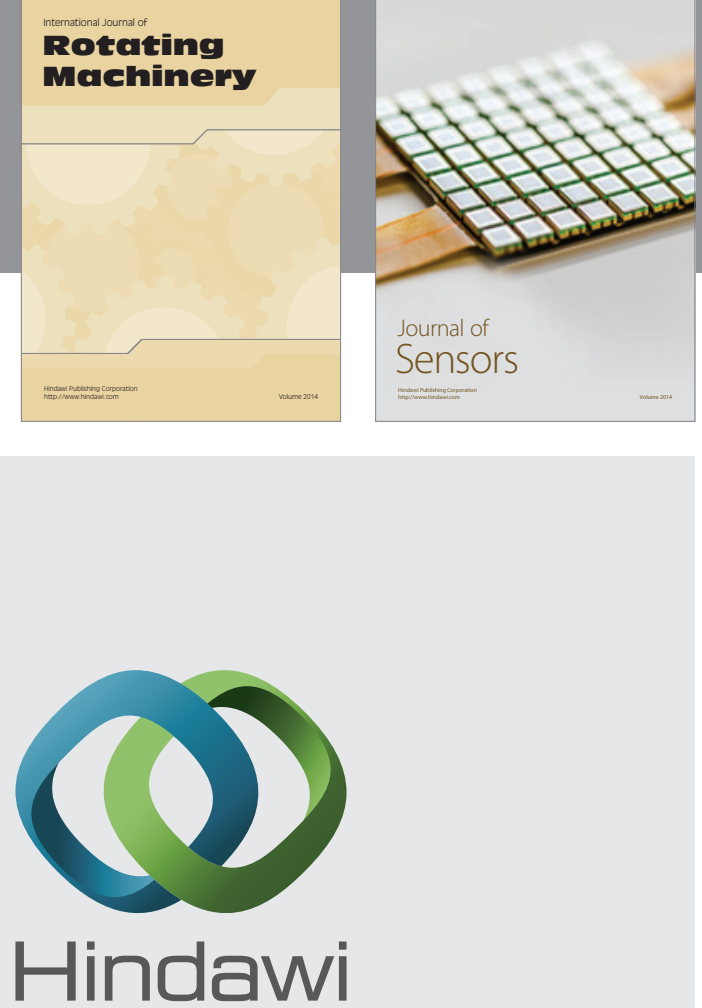

Submit your manuscripts at http://www.hindawi.com
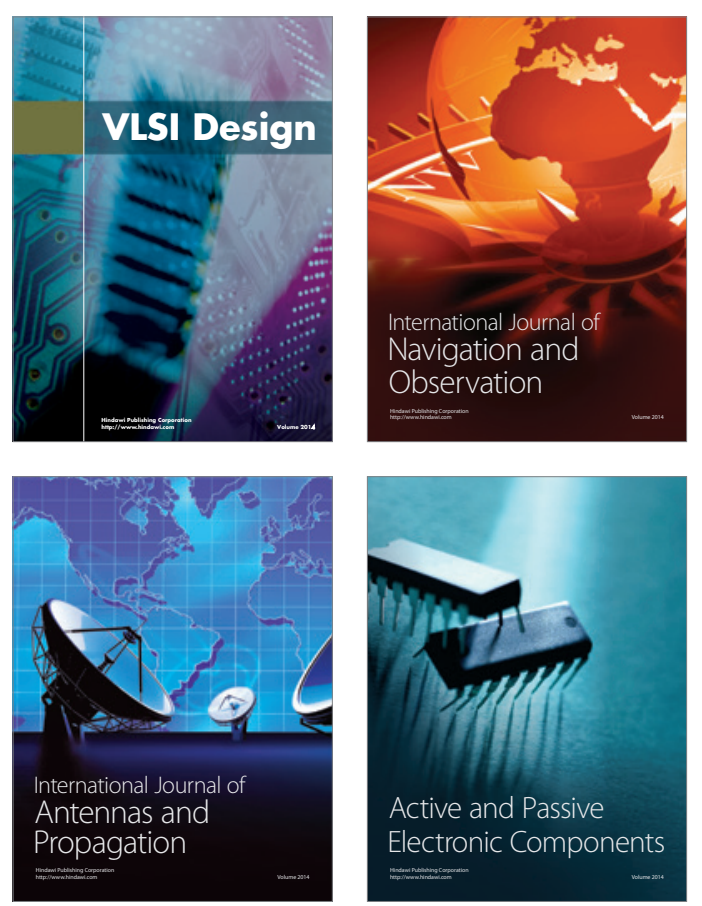


Journal of

Control Science

and Engineering
